\title{
Discounting Future Pain: Effects on Self-Reported Pain
}

\author{
Pablo Brañas-Garza ${ }^{1}$, Maria P. Espinosa ${ }^{2}$, Maria Repolles Pro ${ }^{3}$ \\ ${ }^{1}$ GLOBE: Universidad de Granada, Granada, Spain; ${ }^{2}$ Economics Department, Universidad del País Vasco, Basque, Spain; ${ }^{3}$ Serv. de \\ Anestesiología, Hospital Virgen de las Nieves, Granada, Spain. \\ Email: pbg@ugr.es
}

Received May $13^{\text {th }}, 2010$; revised June $23^{\text {rd }}, 2010$; accepted June $28^{\text {th }}, 2010$.

\begin{abstract}
Empirical results are presented showing that people who acknowledge pain anticipation when expecting an injury experience higher sensitivity to pain (GREP, Robinson et al., 2001). The positive correlation between sensitivity and anticipation is highly significant. However, no relationship is found between anticipation and pain endurance.
\end{abstract}

Keywords: Discounting, Pain Anticipation, Sensitivity and Endurance

\section{Introduction}

"He who suffers before it is necessary, suffers more than is necessary"

Lucius Annæus Seneca (c. 4 BC-65 AD)

Humans are biologically programmed to anticipate threats, a subtle aid to survive the extant challenges in their environment. Recent studies in the field of behavioural economics have shown that people differ in the way they anticipate or value the future (technically, they "discount" the future) in their inter-temporal choices. ${ }^{1}$ Thus, we may find two diametrical personalities: 1) those who are present-oriented and therefore show a more impatient behaviour; and 2) those who are future-oriented and show a self-controlled behaviour.

For instance, people with unhealthy lifestyles (that is, people who smoke, eat junk food, are sport-averse, etc.) are said to seldom highly value the future quality of their lives, thus typifying present-oriented personalities. In other words, they prefer good lives in the present to higher quality of life in the future (see [1]). The common explanation is that fulfilment of their expectations about future lifestyles or events remains highly uncertain due to life's contingencies, and this makes maximizing their current lives more desirable.

Savers typify the future-oriented group. Saving habits provide a good indication of how individuals discount the future. Savers, by keeping part of their current in-

\footnotetext{
${ }^{1}$ Viscusi, Huber \& Bell [2] estimated discount rates and examine how they vary with individual characteristics. See also [3] and [4].
}

come for future use, show that they highly value the future as this deferral is apparently in anticipation of greater benefits accruable from the future use of such income. Thus, they tend to hold a more optimistic view about the fulfilment of their expectations about future lifestyles or events.

Recent research has explored the idea that time perception guides intertemporal choice ([5]). When making decisions, subjects use subjective delays and predict outcomes. Therefore, the subjective time perception regarding the delay of an event affects present behaviour. Thus, those subjects who perceive a shorter delay will be more affected by future events.

Similar arguments may be extended to loss scenarios. For instance, some people may suffer today in anticipation of future worse-case scenarios and anticipatory feelings of future losses or pain affect their present wellbeing. In other words, those who are aware of the likelihood of future pain and perceive a shorter subjective delay could start to "feel" the pain even prior to experiencing the real pain. Thus, anticipation would be more likely for impatient subjects.

This study links pain anticipation and self-reported pain latency. Questionnaires were administered to 122 experimental subjects. Data were collected on individual experience of pain in anticipation of expected injuries and also on pain sensitivity and pain endurance as defined in the Gender Role Expectations of Pain questionnaire (GREP [6]). The subjects reported their pain sensitivity and endurance levels on the Visual Analog Scale 
(VAS), which enabled comparisons with a typical woman and also with a typical man. This task was straightforward and clear to the subjects as it never referred to any specific type of pain, but only to the subject's general attitude towards pain. The results allowed us to measure the subjects' awareness of pain sensitivity and endurance.

The correlation of the GREP measurements with real pain is a critical issue. Defrin et al. [7] showed that pain thresholds and tolerance to real heat pain stimulus correlate significantly with GREP sensitivity measurements but not with endurance ones. Using thermal pain, Wise et al. [8] found that a higher GREP score is associated with both a lower pain threshold and less tolerance to real pain.

Recent literature on pain perception ([9-11]) noted that pain sensitivity and endurance are mediated by socio-cultural factors (e.g. age, ethnicity), psychological factors (e.g. anxiety, depression) and biological factors (e.g. genetics, gonad hormones, endogenous pain inhibitors). Edwards \& Fillingim [12] using a hypothetical test different from GREP, likewise found that self-reported pain sensitivity is uncorrelated with tolerance to real pain but highly correlated with anxiety.

Our paper contributes to the existing literature by advancing the idea that subjects' inter-temporal valuation of the consequences of pain (anticipation of future events) has a nexus with their perception of pain tolerance and endurance. Thus, this research adds a new psychological factor that mediates pain sensitivity.

Our empirical analysis yielded a conclusive result: those who felt pain in anticipation before suffering the real injuries had significantly higher sensitivity values in all categories (that is, with respect to women, men, one's own sex, intersex and the average). In contrast, no differential effect on endurance levels was found. Furthermore, we find that anticipation is related to impatience, which is consistent with the idea of a subjective perception of time.

\section{Research Question}

There is a sequential process following an injury. It begins with pain sensitivity, which refers to the period of time that has elapsed before a person experiences pain after an injury. This is followed by the level of pain endurance, which refers to the period of time that has elapsed before a person experiencing pain will seek relief from their symptoms. These definitions are summarized in Figure 1:

Our hypothesis is that there is an additional step in this sequential process: pain is not only experienced after the

\footnotetext{
${ }^{2}$ There were also many other items not used in this research study, for
} example "propensity to talk to other people about painful experiences." injury, but produces anticipatory discomfort before and immediately after the injury occurs. Once subjects are aware of the future injury, they start to foresee pain and this anticipation shortens the period between injury and pain. Hence, the hypothesis can be formulated as follows: anticipation decreases pain latency. This idea is illustrated in Figure 2.

We conjecture that those who "feel" the pain ex-ante will have shorter pain latency. Hence, we expect sensitivity and anticipation to be positively correlated. Note that anticipation occurs prior to the onset of pain. Hence, endurance should be independent of anticipation.

We test the following hypotheses:

$\mathrm{H1}$ : anticipation and sensitivity are positively correlated.

$\mathrm{H} 2$ : anticipation and endurance are uncorrelated.

A hypothetical experiment was used to check the validity of our hypothesis. The experiment and the dataset are described in the next section.

\section{Data \& Methodology}

\subsection{Variables}

Subjects responded to a two-part questionnaire. Part I was a GREP test (Gender Role Expectation of Pain; $[13,14,6,8])$ in which subjects were requested to report their pain sensitivity and endurance levels on a Visual Analog Scale (VAS). Part II included questions on personal characteristics (age, sex, health, anticipatory behaviour) and social habits related to pain.

\section{Part I. GREP}

Subjects were asked to report their level of sensitivity and endurance as compared to a typical woman and then to a typical man. ${ }^{2}$ The questionnaire was identical to Ro-

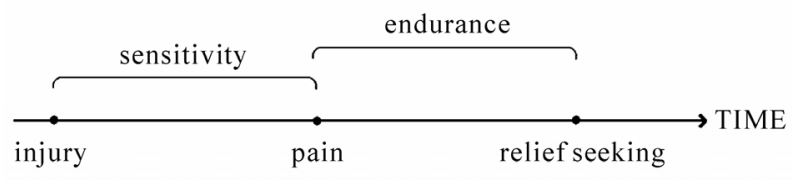

$\left(\mathrm{t}_{\mathrm{i}}\right)$

$\left(t_{p}\right)$

$\left(t_{r}\right)$

Figure 1. Sensitivity and endurance.

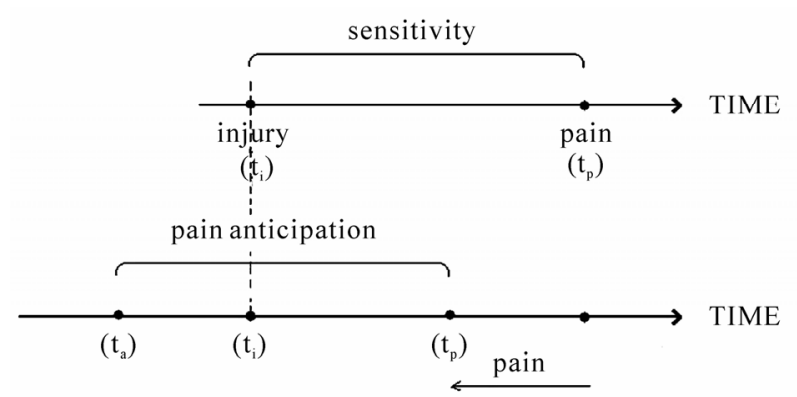

Figure 2. The effect of pain anticipation on sensitivity. 
binson et al. ([13]). ${ }^{3}$ Subjects place a mark on a $10 \mathrm{~cm}$ line to estimate their level of sensitivity and endurance.

The mark is then transformed into an integer number in the interval $[-50,50]$ to measure the subjects' relative position with the center of the interval corresponding to a value equal to the reference point (the typical man or the typical woman). The GREP test provides the following variables:

In Table 1, "same-sex" (sensitivity or endurance) denotes that the comparison is within the responder same sex ( $s_{w}$ and $e_{w}$ for women, and $s_{m}$ and $e_{m}$ for men). "Opposite-sex" denotes that the comparison is made with the other sex ( $s_{m}$ and $e_{m}$ for women, and $s_{w}$ and $e_{w}$ for men).

We also compute the average values, $s_{\text {mean }}=\left(s_{w}+\right.$ $\left.s_{m}\right) / 2$ and $e_{\text {mean }}=\left(e_{w}+e_{m}\right) / 2$. These variables are used to compare each individual to the rest of the society, regardless of gender, and they are obtained to check the robustness of the estimation. However, these average measurements do not have a straightforward interpretation, since sensitivity to pain is gender-related and the typical person is therefore not well defined.

The GREP test is appropriate for our purposes as it captures personal history, that is, the individual compares her own experience (regarding pain) to that of the typical person. The information conveyed refers to the average past pain experiences, presumably of different types, and is therefore not specific to a particular type of pain.

The information obtained from the GREP test is then interpreted as the overall measurement of individual pain sensitivity and endurance. Given previous results by Wise et al. [8] and Defrin et al. [7] regarding the correlation between results in the GREP test and real pain experiments, those who declare themselves to be more sensitive than the average will therefore be considered as having greater sensitivity. Identical argument is used for endurance.

Part II. Pain anticipation and personal characteristics

The question aimed at measuring pain anticipation was formulated as follows: ${ }^{3}$

When you know you are going to suffer a painful experience, do you start feeling pain even before the experience actually takes place? YES $(=1)$ or $\mathrm{NO}(=0)$.

The independent variable, pain anticipation, was derived from this question; $44 \%$ of the subjects stated that they felt pain in advance.

Anticipation could be related to impatience, which is consistent with the idea of a subjective perception of time as the basis for temporal discounting $([5,15])$. In a parallel study, subjects were asked the following hypothetical question: $^{3}$

${ }^{3}$ Translated into Spanish.

${ }^{4}$ Only 57 subjects of this sample also completed the GREP test.
Suppose that we offer you 100 euros that you will receive after 30 days. How much money are you ready to pay in order to get that money tomorrow? That is, which part of the 100 euros you would be willing to sacrifice to get the money in advance? I will pay euros of the 100.

This question was posed to obtain the subjects' level of impatience; 101 subjects complete both the anticipation and the time preference questions. ${ }^{4}$ The amount of money to be paid in order to receive an earlier payment varies substantially across subjects (mean $=14.65 ;$ st. dev. $=17.91 ; \min =0 ; \max =80)$. A simple Pearson test between anticipation (yes/no) and impatience $\left(X^{2}=0.29\right.$; $p$-value $=0.002)$ shows that the distribution of impatience among the subjects who anticipate pain is different from the distribution among those who do not. In particular, subjects who anticipated pain were also willing to pay more to obtain the payment immediately than those who did not. Hence, pain anticipation and impatience are positively correlated.

Participants were also asked to report information on personal characteristics. Age, gender and health status were used as control variables. The descriptive statistics are presented in Table 2.

The health status variable is self-reported; 5 levels are considered, from very bad (0) to excellent (4). Interest-

Table 1. Variables.

\begin{tabular}{ccc}
\hline Original variables & Sens. & Endur. \\
\hline With respect to a typical woman & $\mathrm{s}_{\mathrm{w}}$ & $\mathrm{e}_{\mathrm{w}}$ \\
With respect to a typical man & $\mathrm{s}_{\mathrm{m}}$ & $\mathrm{e}_{\mathrm{m}}$ \\
Transformations & & \\
With respect to a typical person (same sex) & $\mathrm{s}_{\mathrm{same}}$ & $\mathrm{e}_{\mathrm{same}}$ \\
With respect to a typical person (oppos. sex) & $\mathrm{s}_{\mathrm{op}}$ & $\mathrm{e}_{\mathrm{op}}$ \\
Average values & $\mathrm{s}_{\text {mean }}$ & $\mathrm{e}_{\text {mean }}$ \\
\hline
\end{tabular}

Table 2. The descriptive statistics.

\begin{tabular}{cccccc}
\hline & Obs. & Mean & sd. & min & max \\
\hline Age & 122 & 24.40 & 3.19 & 20 & 46 \\
Woman & 122 & 0.55 & 0.49 & 0 & 1 \\
Health & 122 & 2.81 & 0.84 & $1^{*}$ & 4 \\
Anticipation & 122 & 0.44 & 0.49 & 0 & 1 \\
$s_{w}$ & 122 & -9.76 & 23.26 & -50 & 50 \\
$s_{m}$ & 122 & -0.29 & 23.00 & -50 & 50 \\
$e_{w}$ & 122 & 6.90 & 23.15 & -50 & 50 \\
$e_{m}$ & 122 & 0.75 & 22.08 & -50 & 50 \\
\hline
\end{tabular}


ingly, no subject reported the minimum level of health (very bad).

We observe that, on average, subjects declared negative values for $s_{w}$ which indicated that individuals (both males and females) consider themselves less sensitive than the typical woman. This is in contrast with $s_{m}$, where the mean value was not significantly different from zero.

Hence, women are stereotyped as more pain-sensitive than men (as shown in [6]). Identical results were found for pain endurance: on average, both female and male subjects considered their levels of endurance to be higher than that of a typical woman, but not higher than that of a typical man.

\subsection{Experiment and Methodology}

The experiment was conducted in four sessions at the University of Granada (Spain) in September and December 2009. All the sessions were run by the second co-author.

Questionnaire data were collected from 122 university students (55\% women), who voluntarily participated in the experiment. The subjects were Economics and Business students (graduate students and undergraduates in the last two years of their degree). Table 2 reports the main descriptive statistics of the population.

A censored regression model (Tobit) was used to estimate the relationship between pain anticipation and pain sensitivity, on the one hand, and pain anticipation and pain endurance on the other, thereby testing the two main hypotheses. A censored regression or Tobit is similar to a linear model (OLS regression) except that it takes into account that the dependent variable is censored, that is, it cannot take values outside a specified interval. Note that in this study, the values for dependent variables: pain sensitivity and pain endurance were restricted to the interval $[-50,50]$.
The list of independent variables also includes age, gender and health status. The data were processed using STATA10 for MAC.

\section{Results}

Table 3 shows the estimation results for both pain sensitivity and endurance and at the different reference points (typical woman, typical man, typical person of the same sex, typical person of the opposite sex and the average person, respectively). Pain anticipation was significant for pain sensitivity. Subjects who felt pain in anticipation of future injuries also reported higher pain sensitivity values after controlling for sex, general level of health and age.

The bottom part of the table shows the estimation results for pain endurance. In sharp contrast to the pain sensitivity results, pain endurance is not correlated with pain anticipation in all the reference groups.

Hence, pain anticipation appears to be a key psychological factor mediating pain sensitivity. The subjects' inter-temporal valuation of the consequences of pain is related to their perception of pain tolerance. Furthermore, since pain anticipatory behaviour is prior to the onset of pain, we hypothesized that endurance should be uncorrelated to anticipation, which was confirmed by the data.

Figure 3 shows the Box-Plots of pain sensitivity for same sex (on the left) and for opposite sex (on the right) for two groups of subjects, those anticipating pain and those who did not. The Box plots show the first quartile, the median and the third quartile.

We observe that the group of subjects who anticipated (on the right-side of each figure) showed higher values for the three statistics than their colleagues who never anticipated pain (on the left-side of each figure). These results illustrate how pain anticipation can increase the perception of pain sensitivity.

Table 3. Estimation results.

\begin{tabular}{|c|c|c|c|c|c|}
\hline Sensitivity & $s_{w}$ & $s_{m}$ & $S_{\text {same }}$ & $s_{o p}$ & $S_{\text {mean }}$ \\
\hline Age & $0.73(0.28)$ & $-1.67(0.01)$ & $-1.09(0.01)$ & $0.18(0.81)$ & $-0.53(0.33)$ \\
\hline Woman & $12.29(0.00)$ & $10.29(0.01)$ & $-0.11(0.97)$ & $22.96(0.00)$ & $10.73(0.00)$ \\
\hline Health & $4.89(0.05)$ & $1.71(0.49)$ & $1.20(0.57)$ & $5.39(0.06)$ & $3.25(0.12)$ \\
\hline Anticipation & $13.98(0.00)$ & $8.10(0.05)$ & $11.14(0.00)$ & $11.05(0.02)$ & $10.48(0.00)$ \\
\hline Endurance & $\boldsymbol{e}_{w}$ & $e_{m}$ & $e_{\text {same }}$ & $e_{o p}$ & $e_{\text {mean }}$ \\
\hline Age & $1.10(0.11)$ & $-0,26(0.70)$ & $0.08(0.89)$ & $0.76(0.29)$ & $0.46(0.42)$ \\
\hline Woman & $-5.54(0.22)$ & $1.48(0.73)$ & $4.45(0.28)$ & $-8.61(0.07)$ & $-1.88(0.61)$ \\
\hline Health & $1.50(0.56)$ & $-0.85(0.73)$ & $0.24(0.91)$ & $0.41(0.88)$ & $0.28(0.89)$ \\
\hline Anticipation & $2.24(0.61)$ & $2.36(0.57)$ & $5.22(0.20)$ & $-0.70(0.87)$ & $2.44(0.50)$ \\
\hline
\end{tabular}






Figure 3. Pain sensitivity and anticipation

\section{Discussion}

We tested the hypothesis that pain is not only experienced after the injury but produces anticipatory discomfort that shortens the period between injury and pain. Our results indicated that there is a positive relationship between pain sensitivity and pain anticipation, thus suggesting that an anticipatory feeling of pain reduces pain latency by increasing awareness of the future pain.

It may also be argued that the positive correlation between pain anticipation and pain sensitivity is due to the fact that people with lower pain thresholds are more concerned about pain, and therefore likely to suffer more in anticipation of it. However, the fact that pain anticipation and pain endurance are unrelated suggests that higher pain sensitivity is indeed caused by the awareness of the likelihood of future injury.

An interesting question which deserves further analysis is whether the correlation found in the literature between anxiety and pain sensitivity (see [12]) could be related to the psychological factor highlighted in this paper, as individuals who anticipate future pain could also exhibit ensuing anxiety. Finally, a critical issue is how relevant this result is to clinical practice. Previous research has shown that perceptions of pain sensitivity, as measured in the GREP, may predict pain thresholds and tolerance to real pain stimulus ([8] and [7]). Our research suggests that anticipatory behaviour and the resulting perception of pain sensitivity could be useful to predict real pain tolerance and pain thresholds of patients.

\section{Acknowledgements}

Research assistance provided by Antonio Espín and Antonios Proestakis is gratefully acknowledged. Financial support from the Spanish Ministry of Science and Inno- vation (SEJ2007-62081, SEJ2006-06309 and ECO2009 -09120); Basque Government (IT-313-07), the Government of Andalusia Project for Excellence in Research (P07.SEJ.02547) and the Women's Institute of Spain (2008.031) is also gratefully acknowledged.

\section{REFERENCES}

[1] D. Cutler and E. Glaeser, "What Explains Differences in Smoking, Drinking and Other Health-Related Behaviors?" American Economic Review, Vol. 95, No. 2, 2005, pp. 238-242.

[2] W. K. Viscusi, J. Huber and J. Bell, "Estimating Discount Rates for Environmental Quality from Utility-Based Choice Experiments," Journal of Risk and Uncertainty, Vol. 37, No. 2-3, 2008, pp. 199-220.

[3] S. A. Golub, D. T. Gilbert and T. D. Wilson, "Anticipating One'S Troubles: The Costs and Benefits of Negative Expectations," Emotion, Vol. 9, No. 2, 2009, pp. 277-281.

[4] E. Fehr, "Behavioral Science: The Economics of Impatience," Nature, Vol. 415, 2002, pp. 269-272.

[5] M. Wittmann and M. P. Paulus, "Intertemporal Choice: Neuronal and Psychological Determinants of Economic Decisions," Journal of Neuroscience, Psychology and Economics, Vol. 2, No. 2, 2009, pp. 71-74.

[6] M. E. Robinson, J. L. Riley III, C. D. Myers, R. K. Papas, E. A. Wise, L. B. Waxenberg and R. B. Fillingim, "Gender Role Expectations of Pain: Relationship to Sex Differences in Pain," The Journal of Pain, Vol. 2, No. 5, 2001, pp. 251-257.

[7] R. Defrin, L. Shramm and I. Eli, "Gender Role Expectations of Pain is Associated with Pain Tolerance Limit but Not with Pain Threshold," Pain, Vol. 145, No. 1-2, 2009, pp. 230-236.

[8] E. A. Wise, D. D. Price, C. D. Myers, M. W. Heft and M. E. Robinson, "Gender Role Expectations of Pain: Relationship to Experimental Pain Perception," Pain, Vol. 96, No. 3, 2002, pp. 335-342. 
[9] Z. Wiesenfeld-Hallin, "Sex Differences in Pain Perception," Gender Medicine, Vol. 2, No. 3, 2005, pp. 137-145.

[10] S. J. Gibson and M. Farrell "A Review of Age Differences in the Neurophysiology of Nociception and the Perceptual Experience of Pain," The Clinical Journal of Pain, Vol. 20, No. 4, 2004, pp. 227-239.

[11] R. B. Fillingim, C. D. King, M. C. Ribeiro-Dasilva, B. Rahim-Williams, and J. L. Rilley III, "Sex, Gender and Pain: A Review of Recent Clinical and Experimental Findings," The Journal of Pain, Vol. 10, No. 5, 2009, pp. 447- 485.

[12] R. R. Edwards and R. B. Fillingim, "Self-Reported Pain Sensitivity: Lack of Correlation with Pain Threshold and Tolerance," European Journal of Pain, Vol. 11, No. 5,
2007, pp. 594-598.

[13] M. A. Robinson, and E. A. Wise, "Prior Pain Experience: Influence on the Observation of Experimental Pain in Men and Women," The Journal of Pain, Vol. 5, No. 5, 2004, pp. 264-269.

[14] M. E. Robinson, C. Gagnon, J. L. Riley III and D. D. Price, "Altering Gender Role Expectations: Effects on Pain Tolerance, Pain Threshold and Pain Ratings," The Journal of Pain, Vol. 4, No. 5, 2003, pp. 284-288.

[15] K. Kim and G. Zauberman, "Perception of Anticipatory Time in Temporal Discounting," Journal of Neuroscience, Psychology and Economics, Vol. 2, No. 2, 2009, pp. 91101. 